\title{
The Role of Visual and Auditory Information in the Observation and Evaluation of Complex Skills in Gymnastics
}

\author{
Frederike Veit and Thomas Heinen* \\ Faculty of Sport Science, Institute of Movement and Training Science in Sports I, Leipzig University, Germany
}

Submission: March 08, 2019; Published: March 19, 2019

*Corresponding author: Thomas Heinen, Faculty of Sport Science, Institute of Movement and Training Science in Sports I, Leipzig University, Germany

\begin{abstract}
Observing and evaluating motor skills are two activities that are of high importance especially in technical sports such as gymnastics. The question yet arises how these different components are utilized by people of different sport-specific expertise when they observe and evaluate complex gymnastics skills. The study aimed to investigate the role of visual and auditory information in the observation and evaluation of complex skills in gymnastics as a function of sport-specific expertise. Participants with different forms and amount of gymnastics-specific expertise as well as laypeople with no expertise in gymnastics were asked to estimate the duration of the flight phase in straight back somersaults in different experimental conditions. Results of this study support the notion that utilizing visual and auditory information varies as a function of sportspecific expertise in the observation and evaluation of complex skills in gymnastics. For instance, current gymnasts might be more sensitive to information from sources that are more salient during the performance (i.e., auditory information). It can be concluded that emphasizing auditory information can be one beneficial approach for gymnastics training.
\end{abstract}

Keywords: Expert-Novice Paradigm; Common Coding; Paired Comparison; Somersault; Stick-Figure Sequence; Perceptual Sensitivity

\section{Introduction}

Observing and evaluating motor skills are two activities that are of high importance especially in technical sports such as gymnastics. Judges, coaches, and gymnasts use information derived from the observation of motor skills in judging situations, coaching situations, or in modeling situations during motor learning (Jeraj, Veit, Heinen, \& Raab [1], Magill [2]). Information derived from the observation of complex skills comprises first and foremost visual as well as auditory components. The question yet arises how these different components are utilized by people of different sport-specific expertise when they observe and evaluate complex gymnastics skills. Therefore, the study aimed to investigate the role of visual and auditory information in the observation and evaluation of complex skills in gymnastics as a function of sport-specific expertise.

When observing complex skills in sports, people pickup visual as well as auditory information of the observed skills (Hodges \& Williams [3], Magill [2]). On the one hand, visual movement-related information in gymnastics usually comprises the visible performance of skills from a particular perspective of the observer (Lees [4]). On the other hand, auditory movement-related information usually comprises the natural sounds and noises that occur as a result of the interaction of the performer with the environment (i. e., sounds that result from contacting the floor during a reactive leap or during a landing; Kennel, Hohmann, \& Raab [5]). In light of the common coding theory, one could argue that perceived information from both the visual and the auditory system is likely to activate the represented action, while acting, in turn, activates the perceived information (Prinz [6]).

Despite the apparent importance of visual and auditory information in the observation and evaluation of complex skills, first, one could speculate that visual and auditory information are related to different aspects of skill performance. Visual information may be first and foremost related to kinematic variables of gymnastics skill performance (i.e. movement quality, and movement errors; Giblin, Farrow, Reid, Ball, \& Abernethy [7]). Auditory information could be more related to temporal and dynamic variables of the skill, which can hardly be inferred from visual information only, but which might be important in anticipation processes (i. e., reaction forces during contact with the environment, movement rhythm; Allerdissen, Güldenpenning, Schack, \& Bläsing [8], Pizzera \& Hohmann [9]). Second, utilizing and weighting visual and auditory information in the observation and the evaluation on gymnastics skills may depend on the role and experience of the observer as well as on his/her intentions (Jeraj, et al. [1], Pizzera, Möller, \& Plessner [10]). 
Visual information is often considered the most prominent information source (Latash [11], Vickers [12]). However, there is strong evidence that auditory information also plays an essential role in skill observation, anticipation and evaluation (Kennel, Pizzera, Hohmann, \& Schubotz [13], Savelsbergh, Williams, van der Kamp, \& Ward [14]). Cañal-Bruland, Müller, Lach, \& Spence [15] investigated for instance the role of auditory information in the prediction of ball trajectories in tennis. Based on the video-clips of tennis rallies between Novak Đoković and Roger Federer, experienced tennis players were asked to mark the landing position of the ball in the opposite half of the court. The amplitude of the contact sound between the racquet and the ball was systematically manipulated (+/- $12 \mathrm{db})$. Results show that manipulated auditory information had a systematic and significant effect on participant's response behavior. One could, therefore, conclude that auditory information is related to dynamic and temporal aspects in the observation of skill performance and the anticipation of performance outcome.

Sors, Lath, Bader et al. [16] examined volleyball players' prediction performance of overhand serves. The aim was to investigate the impact of (early) auditory and visual information on prediction accuracy. Participants had to anticipate the landing zone of volleyball serves. In a series of three experiments, the authors could show, that the accuracy to predict the landing zone and the length of volleyball overhand serves was significantly higher when auditory information (only) was available compared to when only visual information was available. This result suggests that (early) auditory information include relevant cues to predict volleyball serves, thereby supporting visual information, and leading to a more precise observation and anticipation of motor skill outcomes.

Allerdissen et al. [8] investigated the role of auditory and visual information for action anticipation in fencing. Participant's task was to predict fencing attacks shown as video sequences in a temporal occlusion paradigm in three experimental conditions (visual information only, auditory information only, audio-visual information). The authors could show that motor experts had better anticipation performance than novices in all three experimental conditions. Besides, experts were better in utilizing auditory information when predicting fencing attacks in case no visual information was available. However, experts and novices performed worst in the condition where only auditory information was available. From the results of the study, one could conclude that utilizing and weighting visual and auditory information when observing (complex) skill performance may depend on observers' expertise with the observed skills.

Regarding the studies just mentioned one could assume, that both, visual and auditory information plays an essential role in the observation and evaluation of sports skills. However, in gymnastics, observers might have different amount and kind of expertise with gymnastics skills. Therefore, the question arises how people with different sport-specific expertise utilize visual and auditory information when observing and evaluation complex gymnastics skills. It has been argued, that own motor and visual expertise could improve the visual perception of other people's movements (Loula, Prasad, Harper, \& Shiffrar [17], MacMahon \& Plessner [18]). However, how observers' visual and motor expertise is related to the evaluation of sports skills seems to differ as a function of kind of sports (Pizzera \& Raab [19]). For instance, in ice hockey, it seems that own motor experience might improve judging performance, while this effect could not be found for soccer or handball referees. In addition, visual experience appears to play an important role in the referee's performance in soccer but not in ice hockey (Pizzera \& Raab [19]).

Giblin et al. [7] for instance addressed the question if the motor and perceptual experiences of coaches influence the perception of complex skills in tennis. Participants had to compare two presented serves with regard to perceivable differences in movement kinematics. First, they had to decide, whether observed actions were the same or different. Second, they had to evaluate differences in kinematics between the observed actions. The results showed an increased sensitivity for small kinematic differences. However, it seems that this increase was not based on own movement experiences, which is contradictory to the results of previous studies (Cañal-Bruland, van der Kamp, \& van Kesteren [20], Loffing \& Cañal-Bruland [21]). However, the participant's task was to discriminate rather than to anticipate, which could be one explanation for the contradictory results. One could thus speculate if the results mentioned above generalize to technical sports such as gymnastics where observing small changes in movement kinematics might be of high importance in evaluating complex skill performance.

Pizzera [22] investigated the effect of specific motor experience on perceptual judgments. Judges with specific motor experience and judges without specific motor experience had to evaluate split leaps with leg change on the balance beam. The results show that judges with motor experiences show a better judging performance than judges without motor experiences. Based on the results one could argue that specific motor experience might be associated with judging performance in gymnastics. However, this association seems to be taskdependent (Pizzera, Möller, \& Plessner [10]). Nevertheless, the question remains open which role auditory information plays in gymnastics judging and how this role may vary as a function of gymnastics-specific expertise. Taken together one could argue that both, visual and auditory information plays an essential role in the observation of sports skills. It seems reasonable that auditory information is of high importance in the observation and evaluation of sports skills when the information is related to dynamic and temporal aspects in skill performance determining the performance outcome. As such, auditory information 
seems to support visual information, leading to a more precise observation and evaluation of motor skills. The question yet arises how these different components are utilized by people of different sport-specific expertise when they observe and evaluate complex gymnastics skills. Therefore, the study aimed to investigate the role of visual and auditory information in the observation and evaluation of complex skills in gymnastics as a function of sport-specific expertise. It was hypothesized that the quality of observation and evaluation in gymnastics varies as a function of the available auditory and visual information. It was additionally expected that the quality of observation and evaluation in gymnastics varies as a function of sport-specific expertise with the observed skills.

\section{Material and Methods}

\section{Participants}

In total, $\mathrm{N}=40$ participants were recruited to take part in this study (age range $21-50 ; \mathrm{M}_{\text {age }} \pm \mathrm{SD}=30 \pm 6$ years). The number of participants was derived from a power analysis when expecting a medium effect (Cohen's $\mathrm{f}=0.25$, type-I-error probability $=5 \%$, type-II-error probability $=20 \%$ ). The participants were assigned to one of four groups: 1.) neither motor nor visual expertise with the motor task ( $n_{1}=10$ laypeople $), 2$.) extensive visual expertise with the motor task $\left(n_{2}=10\right.$ judges/coaches $), 3$.) extensive motor expertise with the motor task in the past $\left(\mathrm{n}_{3}=10\right.$ former gymnasts) and 4.) extensive motor expertise with the motor task at the time of the study $\left(\mathrm{n}_{4}=10\right.$ current gymnasts). At the time of the investigation, the laypeople never did gymnastics before, and they had no kind of experience with this type of sport. The judges and coaches had experience in coaching or judging in gymnastics for at least seven years. The former gymnasts were able to perform the experimental task in the past but did not perform the experimental task for the last five years. The current gymnasts were able to perform the experimental task on the floor, and they had at least ten years of experiences in artistic gymnastics. All participants were asked to take part in a study on the observation of gymnastics skills. They were furthermore informed about the procedure and purpose of the study and gave their written consent prior to the beginning of the study, which was in line with the ethical guidelines of the local ethics committee.

\section{Instruments}

Motor Task: The motor task was a sequence of three elements on the floor, namely the round-off, followed by a back handspring, and a straight back somersault (George [23]). The sequence was performed on a standard gymnastics spring floor. A round-off followed by a back handspring and a straight back somersault represents a common acrobatic series in floor exercises (Arkaev \& Suchilin [24]). The straight back somersault should be performed with a maximal flight duration thereby optimizing rotation in to ensure a complete turn about the somersault axis during the flight phase. The round-off and the back handspring serve a preparatory function for the back somersault (i.e., generation of angular momentum towards the take-off; Prassas, Kwon, \& Sands [25]). To prepare stimuli generation for the experiment (see below), ten female expert gymnasts were asked to perform the motor task six times to their best possible performance. Gymnasts performance in the motor task was videotaped with a Full-HD digital video camera operating at $60 \mathrm{~Hz}$, resulting in a total of 60 video sequences. The video camera was placed orthogonal to the movement direction at a distance of about 15 meters from gymnasts' movement plane.

Stimuli Generation: From the total of 60 video sequences, eleven were selected for further stimuli generation. One of the primary criteria was very little background noise together with clear thumping sounds caused by gymnasts contact with the floor when performing the motor task. To reduce surface characteristics and to make it easier for the participants to focus on essential movement-related information, the video sequences were digitized using the software Simi Motion ${ }^{\circledR}$ [26] version 9 (Simi Reality Motion Systems GmbH, Munich, Germany). Coordinates of 14 body landmarks corresponding to the joints of a ten-segment model of the human body were digitized for each frame of the video sequences (Enoka [27]). A stick-figure video sequence was created for each digitized video sequence, comprising a black stick-figure in front of a plain grey background. A straight horizontal black line was inserted into the video sequence representing the floor (see Figure 1 as an example of the motor task as well as of the stimuli format).

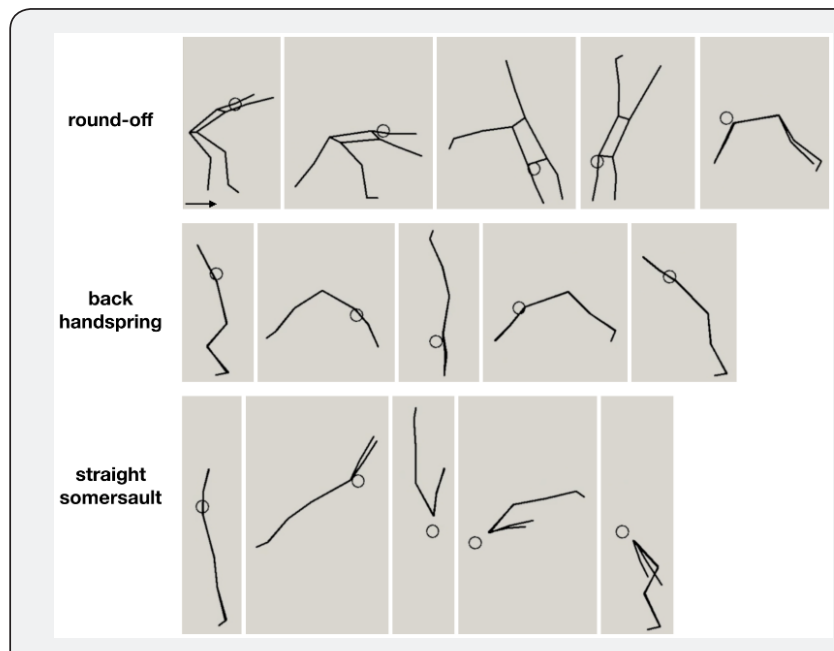

Figure 1: Stick figure sequence of the motor task (round-off, back handspring and straight back somersault).

Experimental Task and Conditions: The experimental task was to observe digitized stick-figure video sequences of the motor task mentioned above. The video sequences were presented on a 27 -inch computer screen in a pairwise fashion, thereby showing one sequence in the upper part of the computer screen and another sequence in the lower part of the computer screen (Effenberg [28] and Kennel et al. [13] for a similar 
approach). Participants task was to estimate which of the two presented stick-figure video sequences comprised the longer flight duration of the straight back somersault. Thus, after watching the video clips the participants had to mark one of the following answers on a prepared questionnaire:

a) Upper video clip showed the longer flight phase.

b) Lower video clip showed the longer flight phase.

c) Upper and lower video clip do not differ concerning the length of the flight phase.

The digitized stick-figure video sequences were presented in one of three experimental conditions:

a) Auditory information only.

b) Visual information only.

c) Audio-visual information. The order of the conditions was randomized for each participant.

Besides, the order of trials within each condition was also randomized for each participant, leading to a total of 165 trials per participant ( $=3$ blocks $x 55$ trials). From participants' choices, the ratio of correct decisions was calculated by summing up the correct answers for each trial in each condition. Participants could achieve a maximum score of 55 and a minimum score of 0 points in each condition.

\section{Procedure}

The study was conducted in three phases. In the first phase, the participant arrived at the laboratory. He/she was informed about the task and the general purpose of the study. Furthermore, the participant was asked to answer some general socio-demographic questions (i.e., gender, age), as well as several additional questions about his/her experiences as a judge/ coach, or gymnast (i.e., years of practice, type of judging/coaching license, etc.). In the second phase, the experiment was conducted. After presenting an example to make sure the participant was calibrated to the task, each trial (video clip) was shown twice on a computer screen. The participant had to mark his/ her choice on a prepared questionnaire. As explained above, the participant could choose between three answers:

a. Upper video clip showed the longer flight phase.

b. Lower video clip showed the longer flight phase.

c. Upper and lower video clip do not differ concerning the length of the flight phase.

The participant was given a short break after every condition. Finally, in the third phase, the participant was debriefed and received a reward for taking part in the study.

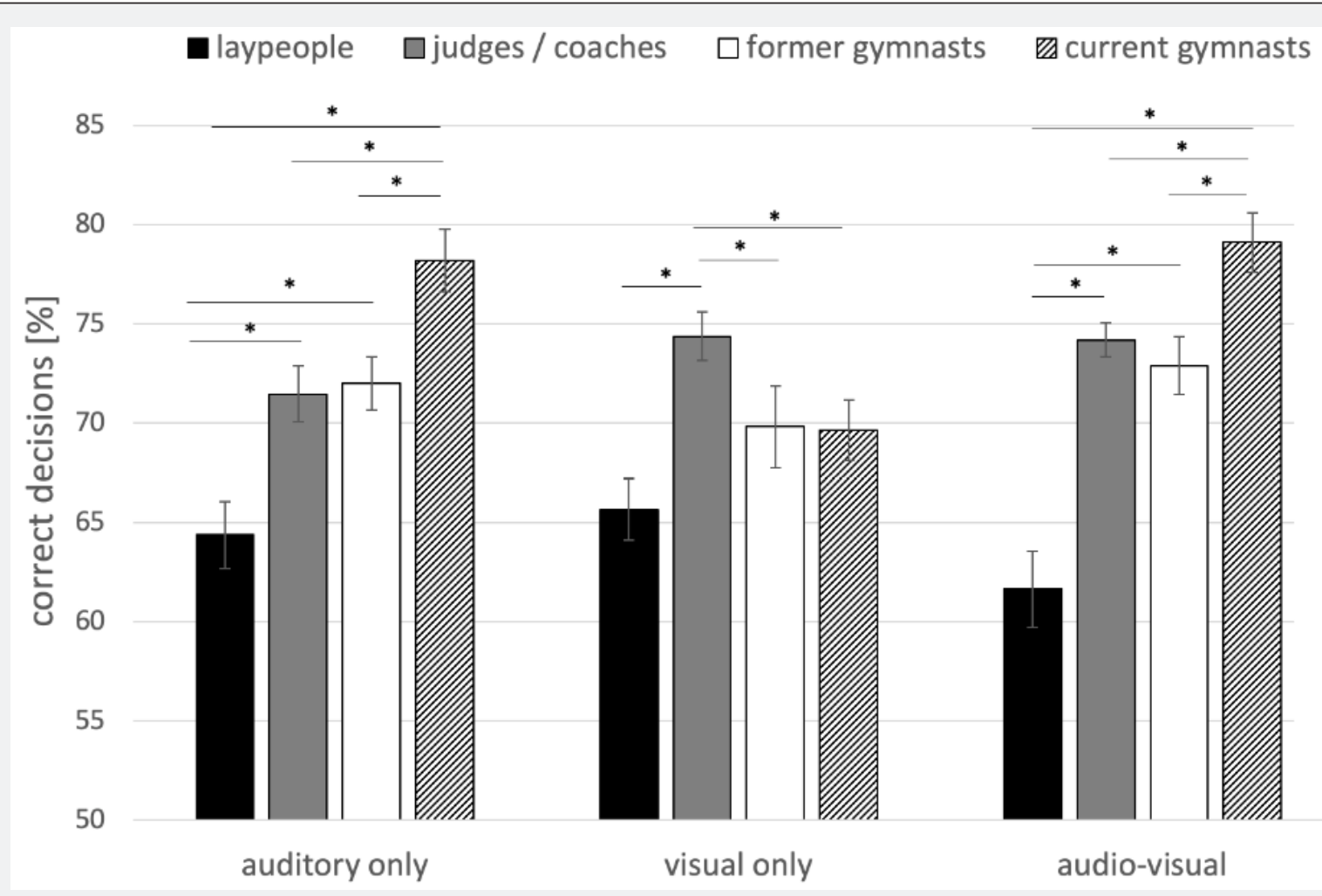

Figure 2: Means and standard errors of participants' relative amount of correct decisions in the experimental conditions. 


\section{Data Analysis}

The level of significance was set at $a=5 \%$ for all results. Statistical analysis was done using SPSS (IBM SPSS statistics, version 23). First, the assumptions to run analyses of variance were examined (normality, sphericity, homogeneity of variances). The results of the Kolmogorov-Smirnov test revealed that the data could be considered to be normally distributed. Results of the Mauchly test showed that the sphericity assumption was not violated. Therefore, no correction of the degrees of freedom was necessary. Furthermore, results of the Levene's-test supported the homogeneity of variances assumption. In order to test the main hypotheses, one 4 (group: 1. laypeople, 2. judges/coaches, 3. former gymnasts, 4 . current gymnasts) $\times 3$ (condition: 1 . auditory information only, 2 . visual information only and 3 . audiovisual information) analysis of variance (ANOVA) was conducted taking the ratio of correct decisions as dependent variable. The factor condition was treated as a within-subject factor, and the factor group was treated as a between-subject factor. Post hoc tests were carried out using Bonferroni's procedure (Knudson [29], Ludbrook [30]). Partial eta square was calculated as an effect size for all significant effects (Cohen [31]). Descriptive statistics are presented as means \pm standard errors (Figure 2).

\section{Results}

It was hypothesized that the quality of observation and evaluation (ratio of correct decisions) varies as a function of the available (visual and auditory) information. Additionally, it was expected that the quality of observation and evaluations varies as a function of sport-specific expertise with the observed skills. In order to test the aforementioned hypotheses, a 4 (group: 1. laypeople, 2 . judges/coaches, 3. former gymnasts, 4 . current gymnasts) $\times 3$ (condition: 1 . auditory information only, 2 . visual information only and 3 . audio-visual information) analysis of variance (ANOVA) with repeated measures was calculated, taking the decision ratio as the dependent variable. The results of the ANOVA revealed a significant main effect of condition on decision ratio, $\mathrm{F}(2,72)=3.298, \mathrm{p}=.043, \eta_{\mathrm{p}}{ }^{2}=.084$, an additional main effect of group on decision ratio, $\mathrm{F}(3,36)=18.628$, $\mathrm{p}<$ $.01, \eta_{\mathrm{p}}^{2}=.608$, and a significant interaction effect of group $\times$ condition on decision ratio, $\mathrm{F}(6,72)=7.210, \mathrm{p}=.0001, \eta_{\mathrm{p}}{ }^{2}=.375$. Participants decision ratio differed between conditions and thus as a function of the available information.

The post hoc tests indicated that participants made significantly more correct decisions in the audio-visual condition than in the visual condition $(\mathrm{p}=.02)$. A non-significant trend was found between the visual only and auditory-only condition $(p=.06)$. Amount of correct decisions differed significantly between groups. More specifically, the ratio of correct decisions of the laypeople was significantly lower than the ratios of the other groups. Moreover, the ratio of the former gymnasts was significantly lower than the ratio of the current gymnasts $(\mathrm{p}=$ .03). Post hoc analysis furthermore supported that laypeople exhibited significant lower ratios of correct decisions in the auditory only and the audio-visual condition compared to the three other groups, while for the visual-only condition, laypeople did not differ from both gymnast groups. Current gymnasts exhibited higher ratios in the auditory and audiovisual condition compared to the three other groups. However, in the visual-only condition, judges and coaches showed a higher ratio compared to the former and current gymnasts. Figure 2 shows the means and standard errors of participants' relative amount of correct decisions in the experimental conditions.

\section{Discussion}

The purpose of the current study was to investigate the role of visual and auditory information in the observation and evaluation of complex skills in gymnastics as a function of sportspecific expertise. Therefore, participants with different forms and amount of gymnastics-specific expertise as well as laypeople with no expertise in gymnastics were asked to estimate the duration of the flight phase in straight back somersaults in different experimental conditions.

Results revealed that participants exhibited a more accurate evaluation of the duration of straight back somersaults in general when both, visual and auditory information was available during observation. As such, auditory information seems to support visual information, leading to a more precise observation and evaluation of gymnastics skills. One could speculate that missing auditory information may hamper the evaluation of complex skills in gymnastics, in particular, if this information is related to dynamic and temporal aspects in skill performance. When performing a somersault, the performance of the reactive leap determines (at least in part) the subsequent flight phase (Prassas et al. [25]). Thus, it could be possible that more information from the reactive leap (visual and auditory information), leads to a more precise evaluation of the flight phase of the somersault. Nevertheless, available visual and auditory information does not simply add up in observing and evaluating gymnastics skills, but instead seem to interact and support each other (Eysenck \& Keane [32]).

Laypeople with no specific expertise in gymnastics performed worse in evaluating the duration of straight back somersaults compared to judges/coaches, former, and current gymnasts. Furthermore, current gymnasts outperformed former gymnasts in the evaluation of the duration of straight back somersaults. These results clearly emphasize that people's' ability to differentiate two gymnastics skills from each other might depend on both, own motor experiences to perform the presented skills, as well as extensive experience watching other people perform the skills (Loula, et al. [17]). It is noteworthy, however, that current gymnasts exhibited a higher ratio of correct decisions than the former gymnasts, thereby indicating that current exposition to gymnastics might even increase the effect of sport-specific experience on observation and evaluation of complex skills. This might at least in part be explained by the idea of shared representations between perception and action 
(Prinz [6]). Performed action is thought to activate represented action-related information. One could speculate that this information is more salient in current gymnasts due to their current exposition to the observed skills in this study, leading to increased perceptual sensitivity (see Knoblich \& Flach [33] for a similar argument).

Finally, the results of this study support the notion that utilizing visual and auditory information varies as a function of sport-specific expertise in the observation and evaluation of complex skills in gymnastics. While current gymnasts may use auditory information to a more substantial degree than participants of the other groups, judges/coaches may use visual information to a more substantial degree than participants of the other groups. Judges and coaches are exposed to gymnastics but spend more time watching and observing skills while current gymnasts spend more time performing skills. Thus, coaches might be more sensitive to the visible performance while current gymnasts might be more sensitive to information from sources that are more salient during the performance. Nevertheless, one could also speculate that judges/coaches and gymnasts differ in their attention when observing and evaluating gymnastics skills. Judges/coaches might in general aim to identify movement errors based on information from body posture that is hardly perceivable based on auditory information only. Judges/coaches do not seem to ignore auditory information because usually a good performance may also comprise a good sound.

There are several limitations of this study, and two particular aspects should be highlighted. First, stick-figure video sequences were used in the experiment as experimental stimuli, in order to control for surface characteristics that could potentially have biased participants decisions (Ste-Marie [34]). One could speculate that laypeople may perceive stick-figure video sequence to some degree unnatural. This, however, should have mainly affected their decision ratio in conditions comprising visual information compared to auditory information. However, laypeople showed lower decision ratios compared to the other groups but not compared across conditions. Nevertheless, it could be of interest to systematically explore the effect of stimuli format on participants' observation and evaluation of complex skills.

Second, the motor task was a sequence of three elements on the floor, namely the round-off, followed by a back handspring, and a straight back somersault. In a back somersault, the goal is to maximize flight duration while optimizing rotation about the somersault axis, so that the gymnast can perform a whole-body rotation in a straight body posture (Prassas, et al. [25]). Based on the results of this study, it seems that auditory information has apparent importance for the current gymnasts when evaluating the flight duration of the somersaults, thereby supporting the results of (Effenberg [28]). However, other skills in gymnastics comprise different movement goals (i. e., circles on the pommel horse or giant swings on high bar), and auditory information might have a different function, and also a different weighting when it comes to observation and evaluation. Thus, it would be of interest to explore whether the results of this study can be generalized to other gymnastic skills that have other movement goals compared to a straight somersault.

\section{Conclusion}

From the results of this study, one could draw conclusions for feedback and modeling procedures in gymnastics. Usually, verbal feedback or visual feedback is provided to the gymnast (Magill [2]). Gymnasts with different amount of motor expertise might utilize and weigh visual and auditory information in a different way. Therefore, it could be most beneficial to either enrich feedback with the information components that a particular gymnast is most sensitive to, or to direct gymnasts' attention to the visual and auditory information components that might be related to skill performance (Baudry, Leroy, Thouvarecq, \& Chollet [35]). This could also be implemented in modeling or imagery procedures (i. e., using an audio-visual model but emphasize the auditory information), thereby helping the gymnast to better progress in motor learning, or to better optimize his/her skill performance. Nevertheless, this effect could differ between skills, because gymnastics skills possess a different amount of interactions between the gymnast and the environment, and thus a different amount of natural sounds occur during the performance. So, for particular elements and element sequences, the usage of a specific combined visualauditory feedback could help gymnasts to implement the corrections.

Additionally, in women's gymnastics floor exercise, music is an inherent component of performance. Given that auditory information might potentially influence (or even bias) peoples' observation and evaluation of gymnastics skill performance, one could speculate about the role that music plays in general in the evaluation of gymnastics skill performance. It could be possible that due to interfering frequencies, natural occurring movement sounds are masked or altered by musical components, leading to a potentially incongruent combination of visual and auditory information. One could easily imagine an intense kick drum sound played back during the reactive leap of a somersault. Even if this does not change the actual flight duration of the somersault, it might potentially bias the perception of the flight duration, which in turn might lead to a biased evaluation of the flight phase duration. Nevertheless, emphasizing or even altering auditory components is a common training intervention (Sors, Murgia, Santoro, \& Agostini [36]).

\section{References}

1. Jeraj D, Veit J, Heinen T, Raab M (2015) How do gymnastic coaches provide movement feedback in training? Int J Sport Sci Coa 10(6): 1015-1024.

2. Magill RA (2007) Motor learning and control. Concepts and applications ( $8^{\text {th }}$ edn). McGraw-Hill, New York, USA.

3. Hodges NJ, Williams AM (Eds) (2012) Skill acquisition in sport. Research, theory and practice. Routledge, London, UK. 
4. Lees A (2002) Technique analysis in sports: a critical review. J Sport Sci 20: 813-828.

5. Kennel C, Hohmann T, Raab M (2014) Action perception via auditory information: agent identification and discrimination with complex movement sounds. J Cogn Psychol 26(2): 157-165.

6. Prinz W (1997) Perception and action planning. Eur J Cogn Psychol 9: 129-154.

7. Giblin G, Farrow D, Reid M, Ball K, Abernethy B (2016) Does perceptual or motor experience influence the perception of global and jointspecific kinematic changes in complex movement patterns? Atten Percept Psycho 78(6): 1781-1793.

8. Allerdissen M, Güldenpenning I, Schack T, Bläsing B (2017) Recognizing fencing attacks from auditory and visual information: a comparison between expert fencers and novices. Psychol Sport Exerc 31: 123-130.

9. Pizzera A, Hohmann T (2015) Acoustic information during motor control and action perception. A review. The Open Psychology Journal 8: 183-191.

10. Pizzera A, Möller C, Plessner H (2018) Gaze behavior of gymnastic judges: where do experienced judges and gymnasts look while judging? Res Q Exercise Sport 89(1): 112-119.

11. Latash ML (2008) Neurophysiological basis of movement ( $2^{\text {nd }}$ edn). Human Kinetics, Champaign, IL.

12. Vickers JN (2007) Perception, cognition, and decision training: the quiet eye in action. Human Kinetics, Champaign, IL.

13. Kennel C, Pizzera A, Hohmann T, Schubotz RI (2014) The perception of natural and modulated movement sounds. Perception 43(8): 796-804.

14. Savelsbergh GJP, Williams AM, van der Kamp J, Ward P (2002) Visual search, anticipation and expertise in soccer goalkeepers. J Sport Sci 20(3): 279-287.

15. Cañal-Bruland R, Müller F, Lach B, Spence (2018) Auditory contributions to visual anticipation in tennis. Psychol Sport Exerc 36(1): 100-103

16. Sors F, Lath F, Bader A, Santoro I, Galmonte A (2018) Predicting the length of volleyball serves: the role of early auditory and visual information 13(2): e0208174.

17. Loula F, Prasad S, Harper K, Shiffrar M (2005) Recognizing people from their movement. J Exp Psychol 31(1): 210-220.

18. MacMahon C, Plessner H (2008) The sport official in research and practice. In: D Farrow, J Baker, C MacMahon (Editors), Developing sport expertise: researchers and coaches put theory into practice. Routledge, New York pp. 172-192.

19. Pizzera A, Raab M (2012) Perceptual judgments of sports officials are influenced by their motor and visual experience. J Appl Sport Psychol 24: 59-72.
20. Cañal-Bruland R, van der Kamp J, van Kesteren J (2010) An examination of motor and perceptual contributions to the recognition of deception from others' actions. Hum Mov Sci 29(1): 94-102.

21. Loffing F, Cañal-Bruland R (2017) Anticipation in sport. Curr Opin Psychol 16: 6-11.

22. Pizzera A (2012) Gymnastic judges benefit from their own motor experience as gymnasts. Res Q Exercise Sport 83(4): 603-607.

23. George GS (2010) Championship gymnastics: biomechanical techniques for shaping winners. Designs for Wellness Press, Carlsbad, CA.

24. Arkaev LI, Suchilin NG (2004) How to create champions. The theory and methodology of training top-class gymnasts, Meyer \& Meyer Sport, Oxford, UK.

25. Prassas S, Kwon YH, Sands WA (2006) Biomechanical research in artistic gymnastics: a review. Sport Biomech 5(2): 261-291.

26. Simi Motion ${ }^{\circledR}$ (Version 9) [Computer software]. Munich, Germany: Simi Reality Motion Systems GmbH

27. Enoka RM (2008) Neuromechanics of Human Movement ( $4^{\text {th }}$ edn), Human Kinetics, Champaign, IL.

28. Effenberg A (2005) Movement sonification: Effects on perception and action. IEEE Multi Media 12(4): 53-59.

29. Knudson D (2009) Significant and meaningful effects in sports biomechanics research. Sport Biomech 8(1): 96-104.

30. Ludbrook J (1998) Multiple comparison procedures updated. Clin Exp Pharmacol P 25: 1032-1037.

31. Cohen J (1988) Statistical power analysis for the behavioral sciences ( $2^{\text {nd }}$ edn), Lawrence Erlbaum Associates, Hillsdale, NJ.

32. Eysenck MW, Keane MT (2010) Cognitive psychology. A student's handbook ( $6^{\text {th }}$ edn), Psychology Press, Hove, East Sussex, UK.

33. Knoblich G, Flach R (2001) Predicting the effects of actions: interactions of perception and action. Psychol Sci 12(6): 467-472.

34. Ste-Marie DM (2003) Expertise in sport judges and referees. Circumventing information-processing limitations. In: JL Starkes, KA Ericsson (Editors.), Expert performance in sports: advances in research on sport expertise. Human Kinetics, Champaign, IL pp. 169-190.

35. Baudry L, Leroy D, Thouvarecq R, Chollet D (2006) Auditory concurrent feedback benefits on the circle performed in gymnastics. J Sport Sci 24(2): 149-156.

36. Sors F, Murgia M, Santoro I, Agostini T (2015) Audio-based interventions in sport. The Open Psychology Journal 8: 212-219.

\begin{tabular}{|l|}
\hline Your next submission with Juniper Publishers \\
will reach you the below assets \\
- Quality Editorial service \\
- Swift Peer Review \\
- Reprints availability \\
- E-prints Service \\
- Manuscript Podcast for convenient understanding \\
- Global attainment for your research \\
- Manuscript accessibility in different formats \\
( Pdf, E-pub, Full Text, Audio) \\
- Unceasing customer service \\
Track the below URL for one-step submission \\
https://juniperpublishers.com/online-submission.php \\
\end{tabular}

